\title{
A Virtual Environment for the Simulation of 3D Wood Strands in Multiple View Systems for the Particle Size Measurements
}

\author{
Ruggero Donida Labati, Angelo Genovese, Vincenzo Piuri, Fabio Scotti \\ Department of Computer Science \\ Università degli Studi di Milano \\ Milano, 20122, Italy. \\ \{ruggero.donida, angelo.genovese, vincenzo.piuri, fabio.scotti\}@unimi.it
}

\begin{abstract}
In this paper, we present a complete virtual environment for the computation of synthetic three-dimensional samples representing free falling wood strands.The proposed method permits to simulate acquisitions performed by real multiple view setups in which the stream of strands falling out of a conveyor belt is analyzed with image processing techniques in order to compute the particle size distribution. Unfortunately, experiments in real time applications are complex and expensive, and the ground true is almost impossible to measure in such conditions. The creation of a metric and fully virtual environment of falling wood strands represent a key feature in order to properly design the illuminotecnic and optical setups, optimize the image processing methods as well as the three- dimensional reconstruction techniques, using controlled and fully repeatable virtual image datasets.

The implemented method first computes realistic threedimensional models of wood strands with known shape characteristics. Then, the strands are rotated and translated into a global three-dimensional space. Finally, realistic effects are applied and images related to different viewpoints are computed.

Experimental results show that the realized virtual environment can effectively be used for the simulation of multiple view acquisitions, obtaining realistic synthetic samples and effectively allowing designing and test image processing algorithms.
\end{abstract}

\section{INTRODUCTION}

In the wood panel production, the size measurement of the wood strands and chips composing the boards (Fig. 1 a) is a task of great importance and it is directly related to the quality of the final product. The measurement process is usually performed offline by using laboratory instruments, which are typically based on the sieving technique [1]. Measurements based on this technique, however, are time consuming and can obtain limited accuracy. In order to overcome these limitations, methods based on image analysis have been studied [2-5]. Another important advantage of image processing techniques is that they can be applied inline.

The design of inline systems for the strand size measurements is particularly complex since it has to consider different non-idealities. The analyzed particles, in fact, can present occlusions and important differences in size and orientation. Moreover, they have irregular shapes which cannot be properly approximated to spheres or polygons in order to easily estimate the particle size distribution of the productive process [6]. (a)
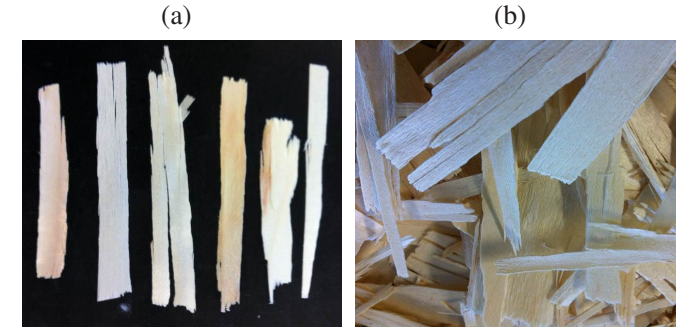

Fig. 1. Examples of real wood strand images captured in our laboratory with a CCD camera in natural light conditions: (a) wood strands posed on a black surface; (b) wood strand mat.

Another aspect that should be considered consists in the need of real-time methods able to analyze big amounts of material. The presence of dust and poor illumination conditions are other problems that should be addressed. As an example of the problem complexity, Fig. 1 b shows an image of a wood strand mat created in our laboratory.

The implementation and evaluation of both two-dimensional and three-dimensional methods for the size measurement of wood strands and chips require the use of big datasets of images describing particles with known size properties. The acquisition of these datasets, however, can be complex and expensive. Accurate measurements of the single particles, in fact, are time consuming and cannot be performed in all the steps of the productive process. Moreover, the measurement procedures can modify the shape of the particles due to their fragility, reducing the number of elements that can be used to test the measurement system. During the design of vision systems, it should also be necessary to modify the acquisition setups. This task can be particularly complex in inline applications since it can require modifying other mechanical components of the productive line. Methods for the computation of realistic synthetic samples describing particles with known shape characteristics should therefore be useful to save time and money during the design of systems for the quality control of the wood panel production.

At the best of our knowledge, in the literature there are only studies on methods for the computation of synthetic two-dimensional images of wood particles [7,8]. Simulation 


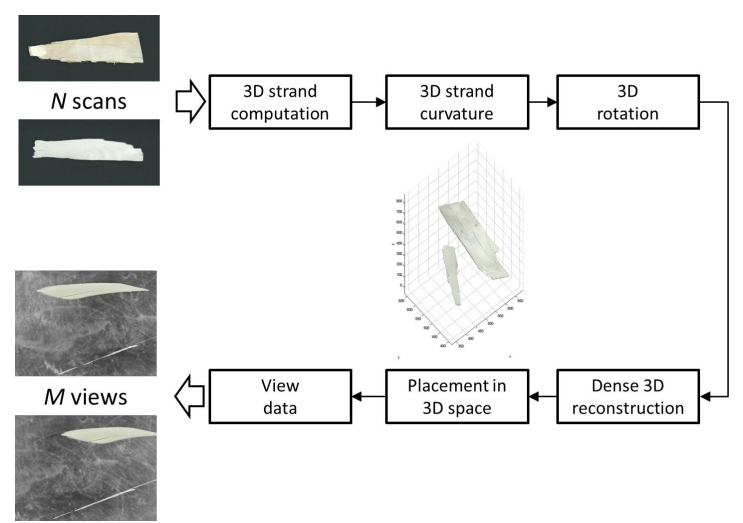

Fig. 2. Schema of the proposed method. Starting form $N$ acquisitions of wood strands achieved by a consumer scanner, the reconstruction of $M$ realistic views of the scene is performed simulating real environments that can be found along the wood panel production line (e.g. falls of strands and layers on conveyor belts) also in terms of background, illumination conditions, scattering in the air due to dust and camera noise.

methods based on three-dimensional data are only used to analyze the quality of the productive process and material [9], and cannot be used to design and test measurement systems based on image analysis techniques.

In this paper, we propose a complete virtual environment for the computation of synthetic three-dimensional samples describing sets of free falling wood strands. The proposed virtual environment is designed to be used for the implementation and test of measurement systems based on image processing and multiple view acquisition techniques. The obtained samples have known size characteristics and can therefore permit to easily evaluate the accuracy of measurement algorithms based on image processing methods.

For each simulated strand, the implemented method uses previously performed scans in order to compute threedimensional point clouds describing every face of the particle. Then, shape irregularities are applied to every threedimensional model. The simulated strands are then randomly rotated an translated in the three-dimensional space. The position of every three-dimensional strand is controlled by using collision detection techniques. Finally, images related to one or more views are simulated by considering the intrinsic and extrinsic parameters obtained by calibrated real acquisition setups. The schema of the method is shown in Fig. 2.

The paper is structured as follows. Section II describes related works on methods for the measurement and simulation of wood strands and chips. Section III presents the proposed method. In Section IV, the experimental results are detailed. Finally, Section V summarizes the work.

\section{RELATED WORKS}

The quality of wood panels is related to different aspects of the productive process. In order to obtain good quality products, it is therefore necessary to monitor all the steps of the process. In many of these steps, the quality of the production is inferred by measuring the size of strands and chips.

With respect to traditional measurement systems based on the sieving technique [1], methods based on image processing algorithms permit to reduce the measurement time and can also be applied inline. These systems are based on different acquisition techniques and are designed for different applicative scenarios.

One of the first studies on automatic image analysis techniques for the measurement of wood particles is described in [2] and performs the image acquisition by using a sonic $x-y$ digitizer. Image analysis methods based on scanner acquisitions are also used in [3] to evaluate the relation between the shape of the strand and mechanical properties of the final OSB panels. Another study on the quality of wood strands used for the OSB production is presented in [4]. The acquisition setup consists in a CCD camera and two light sources. The captured samples consist in images of small sets of strands manually disposed on a reference plan. The method described in [5] uses image analysis algorithms and computational intelligence classifiers to measure the particle size distribution in images of wood strand mats.

Other methods in the literature are based on threedimensional data acquired by using laser scanners [10] and consider wood strands disposed on conveyor belts.

The design and testing of measurement systems based on image processing techniques require big sets of samples describing wood particles with known shape characteristics. In order to reduce the time and cost of the sample acquisition and preliminary measurement process, techniques for the computation of synthetic data have been researched. A study on the particle size measurement that uses simple synthetic images is described in [8]. Synthetic data have also been successfully used to increase the accuracy of computer vision systems designed for different applicative contexts $[11,12]$.

In the literature, there are techniques for the simulation of the distribution of three-dimensional strands during the productive process [9]. These techniques, however, do not simulate visual data but only compute volume information in order to analyze the production and properties of wood-based composite materials.

A method for the computation of synthetic images describing different kinds of particles is presented in [7]. This technique can obtain realistic results but does not permit to simulate images of samples with known shape characteristics captured by real multiple view systems.

There are also studies on the simulation of free falling particles, like snow [13] and sand [14].

More general methods for the computation of threedimensional objects are described in $[15,16]$.

\section{THE PROPOSED METHOD}

In this paper, we present a complete virtual environment for the three-dimensional simulation of free falling wood strands designed to be used for the implementation and test of measurement systems based on image processing and multiple view acquisition techniques. The simulated particles have known shapes and can therefore permit to easily evaluate the accuracy of measurement algorithms. Moreover, the obtained 
images present high visual realism since they are based on textures obtained from real acquisitions.

Starting from $N$ scans of real strands, the method therefore computes a three-dimensional model of a virtual space and then it renders $M$ images representing $N$ simulated wood strands acquired by $M$ cameras of a real acquisition system. The method can be divided into the sequent steps (Fig. 2):

1) $3 \mathrm{D}$ strand computation;

2) 3D strand curvature;

3) 3D rotation;

4) dense $3 \mathrm{D}$ reconstruction;

5) placement in 3D space;

6) view data.

The last steps of the proposed method can be realized by using software applications designed for the render of threedimensional scenes (e.g. Blender [17] and Autodesk 3DS MAX [18]). However, we have decided to implement the proposed virtual environment as an executable software since an important goal of this study is to provide a tool usable by untrained employees, which does not require third party applications and can be portable to different computational architectures. The implemented virtual environment, in fact, should also be used together with real data for the continuous training of measurement systems in real productive scenarios.

\section{A. 3D strand computation}

The first step of the proposed method consists in the simulation of the three-dimensional shape of $N$ wood strands. For each strand, a scanned image $I$ and the measured thickness $h$ are used to obtain a three-dimensional representation of the wood particle. The three-dimensional model is considered as composed by two surfaces with arbitrary shapes. Two point clouds $C_{1}$ and $C_{2}$ and two textures $T_{1}$ and $T_{2}$ are therefore computed to obtain a representation of the particle.

The two-dimensional shape of the strand is first estimated by computing the region of interest (ROI) of $I$ with the Otsu's method [19]. An example of image $I$ and the corresponding ROI are shown in Fig. 3 a and Fig. 3 b, respectively.

The vectors $X_{i}$ and $Y_{i}$ describing the coordinates $\left(x^{\prime}, y^{\prime}\right)$ of the point clouds $C_{1}$ and $C_{2}$ are obtained by converting in millimeters the pixel coordinates $(x, y)$ of every point $j$ appertaining to the ROI:

$$
\begin{aligned}
& X_{i}(j)=x(j)(r / 25.4), \\
& Y_{i}(j)=y(j)(r / 25.4),
\end{aligned}
$$

where $r$ is the scanner resolution expressed in DPI.

A first approximation of the coordinates $z^{\prime}$ of the point clouds $C_{1}$ and $C_{2}$ is then computed for each pixel $j$ appertaining to the ROI:

$$
\begin{aligned}
& Z_{1}(j)=0, \\
& Z_{2}(j)=h .
\end{aligned}
$$

In order to approximate the lateral shape of the strand, the boundaries of the three-dimensional model are then closed in the three-dimensional space. The boundary of the ROI is searched by using morphological operators, and the $z^{\prime}$ coordinates corresponding to every pixel $b$ appertaining to the boundary are then modified as:

$$
\begin{aligned}
& Z_{1}(b)=h / 2, \\
& Z_{2}(b)=h / 2 .
\end{aligned}
$$

The three-dimensional coordinates of every pixel $b$ appertaining to the boundary are then stored in the point cloud matrix $B$ in order to simplify subsequent rendering tasks.

An example of obtained strand model is shown in Fig. 3 c.

\section{B. $3 D$ strand curvature}

This step aims to increase the realism of the simulated threedimensional particles by inserting curvatures of the surfaces that are typically present in real wood strands due to the cutting process.

A first curvature is computed by considering the $y$ and $z$ axes. A polynomial $p_{z}$ approximating the curvature is defined as the second order polynomial passing by the $y$ and $z$ coordinates $\left(\min \left(Y_{i}\right), 0\right),\left(\left(\min \left(Y_{i}\right)+l_{y} / 3+\operatorname{rand}\left(l_{y} / 3\right), \operatorname{rand}\left(c_{z}\right)\right)\right.$, $\left(\max \left(Y_{i}\right), 0\right)$. The function $\operatorname{rand}()$ computes a random number between 0 and threshold value, $l_{y}=\max \left(Y_{1}\right)-\min \left(Y_{i}\right)$, and $c_{z}$ is an empirically estimated constant.

A vector $Z_{c}$ representing the curvature is obtained by fitting the polynomial $p_{z}$ in the interval from $\min \left(Y_{i}\right)$ to $\max \left(Y_{i}\right)$.

Every column $j$ of every depth vector $Z_{i}$ is then defined as:

$$
Z_{i}(j)=Z_{i}(j)+Z_{c}
$$

A curvature computed considering the $x$ and $z$ axes is also inserted in a similar manner.

The curvatures are then applied to the boundary matrix $B$.

Fig. $3 \mathrm{~d}$ shows an example of obtained point cloud.

\section{C. $3 D$ rotation}

A random rotation in the three-dimensional space is applied to the point clouds $C_{1}$ and $C_{2}$. The rotated point clouds are computed by using rigid transformations:

$$
C_{i}=C_{i} R_{x}(\phi) R_{y}(\theta) R_{z}(\psi),
$$

where $R_{x}(\phi), R_{y}(\theta), R_{z}(\psi)$ are rotation matrices describing a rotation in the $x, y$, and $z$ directions, respectively. The values $\phi, \theta$, and $\psi$ are random numbers in the range from 0 to $2 \pi$.

The boundary matrix $B$ is also rotated by using the same parameters.

An example of rotated three-dimensional model of a wood strand is shown in Fig. 3 e.

\section{Dense $3 D$ reconstruction}

This step computes a voxel representation of the threedimensional strand in order to easily permit a subsequent placement of the simulated particle in a three-dimensional reference space and a dense rendering of the data.

For each point cloud $C_{i}$ of a strand, the two-dimensional matrices $X_{D i}, Y_{D i}$, and $Z_{D i}$ representing the voxel coordinates of a dense three-dimensional reconstruction are computed. The matrices $X_{D i}$ and $Y_{D i}$ represent the coordinates of the plane $\left(\min \left(X_{i}\right) \ldots \min \left(X_{i}\right), \min \left(Y_{i}\right) \ldots \min \left(Y_{i}\right)\right)$ with 
(a)

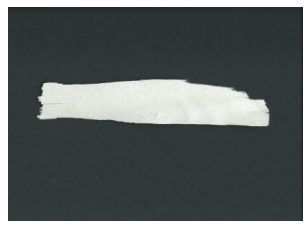

(c)

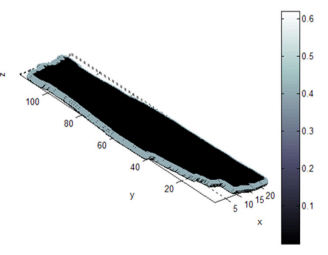

(e)

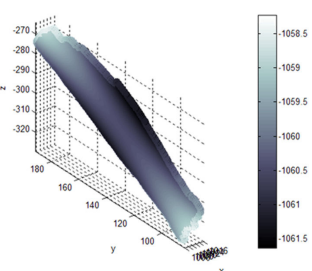

(b)

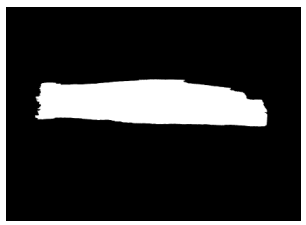

(d)

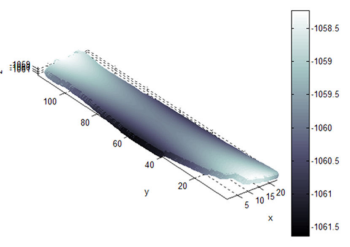

(f)

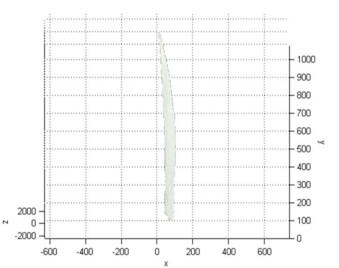

Fig. 3. Computation of a dense three-dimensional model of a single strand: (a) scanned image $I$; (b) region of interest (ROI); (c) point clouds $C_{1}$ and $C_{2}$; (d) $C_{1}$ and $C_{2}$ after the strand curvature; (e) $C_{1}$ and $C_{2}$ after a random threedimensional rotation; voxel representations $D_{1}$ and $D_{2}$ with superimposed textures $T_{D} 1$ and $T_{D} 2$.

a constant step $s$. The depth map $Z_{D i}$ is then obtained by applying a bilinear interpolation of the points appertaining to $P_{i}$ in the coordinates of the matrices $X_{D i}$ and $Y_{D i}$. The values of $Z$ are then discretized with step $s$.

The interpolation step introduce artifacts in the border of the depth map. For this reason, the region of interest of the map $Z_{D i}$ is limited to the points appertaining to the binary mask $B$, which is computed as the area appertaining to the boundary coordinates $B_{i}$ in the voxel space.

A similar procedure is applied to computate the texture $I_{D i}$.

An example of the obtained results is shown in Fig. $3 \mathrm{f}$.

\section{E. Placement in $3 D$ space}

In this step, $M$ strands are randomly placed in a reference three-dimensional space $S(x, y, z)$ with coordinates $x \in$ $\left.\left[x_{\min } \ldots x_{\max }\right], y \in\left[y_{\min } \ldots y_{\max }\right], z \in\left[z_{\min } \ldots z_{\max }\right)\right]$. The limits of the three-dimensional space can be changed for each simulation, and are related to the considered setup.

In order to avoid possible placement errors, a collision detection algorithm is applied before positioning every wood strand $S j$, which is considered as composed by the points appertaining its surfaces $D_{q}$ and $D_{2}$. This method checks if the wood strand $S j$ shares common voxels with the previously positioned $j-1$ three-dimensional objects. If a collision is detected, the coordinates of the model $j$ are recomputed.

The first task of the collision detection strategy aims to reduce the computational time and evaluates if the bounding spheres of two particles can collide. The center coordinates

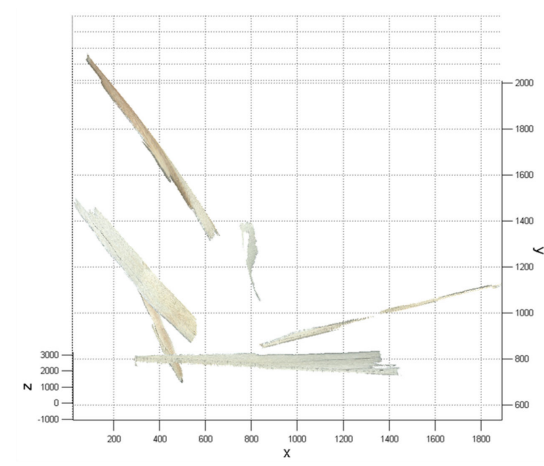

Fig. 4. Example of wood strands disposed in the voxel space $S$.

$\left(x_{j}, y_{j}, z_{j}\right)$ of a bounding sphere are obtained as the mean coordinates of $S_{j}$. The radius $r_{j}$ is defined as

$$
r_{j}=\max \left(l_{j 1}, l_{j 2}, l_{j 3}\right),
$$

where $l_{j 1}, l_{j 2}, l_{j 3}$ are the lengths of the dimensions of $S_{j}$.

A possible collision is detected if $d_{j k}<r_{j}+r_{k}$. The value $d_{j k}$ is the Euclidean distance between the center coordinates.

If a possible collision is dected, a second control is applied. Two three-dimensional matrixes $V_{j}$ and $V_{k}$ representing the voxel spaces of the considered three-dimensional models are computed. In these matrixes, elements equal to 1 represent occupied voxels, and elements equal to 0 represent empty voxels. A matrix describing the collided voxels is then computed as

$$
V(x, y, z)=V_{j}(x, y, z) \wedge V_{k}(x, y, z) .
$$

A collision is finally detected if there is at least one element of $V$ equal to 1 .

An example of wood strands disposed in the voxel space $S$ is shown in Fig. 4.

\section{F. View data}

The final step of the proposed method computes $M$ images representing the acquisitions performed by $M$ cameras.

In order to obtain the first frontal view, $2 N$ images $I_{i}$ representing the $3 \mathrm{D}$ mapping of every surface $D_{i}$ are computed. First, the two-dimensional coordinates $p$ of every threedimensional point $P$ are obtained as projection based on the intrinsic parameters $A$ related to a real calibrated camera [20].

Similarly to the dense 3D reconstruction step, a bilinear interpolation in a space $\left(1 \ldots, i_{x}, 1 \ldots, i_{y}\right)$ is applied to obtain the graphical representation of every image $I_{i}$.

The final image $I_{F}$ captured by the frontal camera is then obtained by merging the regions of every image $I_{i}$ with the lowest distance from the camera.

In order to properly simulate the other $M-1$ views of the acquisition system, a rigid transformation is applied to every three-dimensional point cloud $C_{i}$ as:

$$
C_{i}^{\prime}=R \cdot C_{i}+T,
$$

where $R$ and $T$ are the rotation and translation matrices obtained from a calibration of a real multiple view system [20]. Finally, a dense 3D reconstruction is computed and the implemented 3D mapping strategy is applied. 


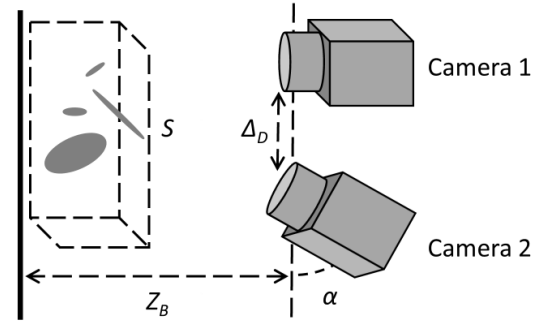

Fig. 5. Simulated acquisition setup.

(a)

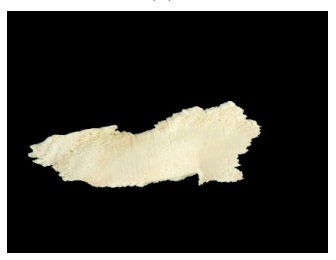

(c)
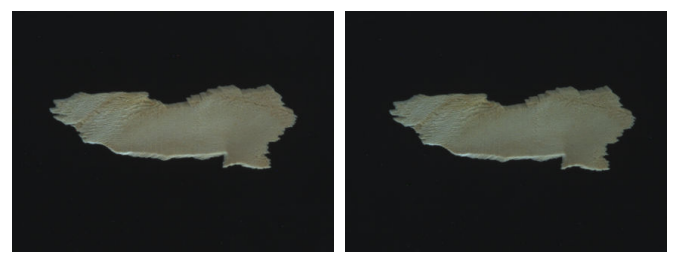

Fig. 6. Simulated and real images related to the same acquisition setup: (a) simulated image of Camera 1; (b) simulated image of Camera 2; (c) real image of Camera 1; (d) real image of Camera 2.

\section{EXPERIMENTAL RESULTS}

In order to evaluate the proposed method, we have collected a dataset of 80 scanned images representing single wood strands. The images have a resolution of 300 DPI. For every captured image, we have also measured the thickness $h$ of the particle center with a precision of $\pm 0.05 \mathrm{~mm}$.

Using these samples, we have created synthetic data related to different hardware configurations. We have considered the real acquisition setup shown in Fig. 5.

The used setup parameters are $\alpha=10^{\circ}, \Delta_{D}=85 \mathrm{~mm}$, space $S$ with coordinates $x \in[-75 \mathrm{~mm} \ldots 75 \mathrm{~mm}], y \in$ $[-75 \mathrm{~mm} \ldots 75 \mathrm{~mm}], z \in[350 \mathrm{~mm} \ldots 640 \mathrm{~mm}]$, and $Z_{B}=$ $700 \mathrm{~mm}$. The image parameters are $i_{x}=1280$ pixel and $i_{x}=960$ pixel. The intrinsic camera parameters are related to Sony XCD-SX90CR CCD color cameras with focal length of $50 \mathrm{~mm}$. The voxel size is $s=0.1 \mathrm{~mm}$.

The first performed test consists in the comparison of simulated and real samples of single strands with the same hardware configurations. Considering that it is difficult to place wood strands with known three-dimensional orientations in real applications, we compared particles placed on a black reference surface. A pair of simulated images and the corresponding real acquisitions are shown in Fig. 6. It is possible to observe that real and simulated images are very similar. The most important difference consists in the used illumination.

Moreover, we compared the convex hull area of the region of interest of real and simulated acquisitions. The results
TABLE I

CONVEX HULL AREA COMPUTED FROM REAL AND SIMULATED SAMPLES.

\begin{tabular}{lccr}
\hline \hline & \multicolumn{2}{c}{ Convex hull area (pixel) } & \\
& Real acquisition & Simulated acquisition & Error (\%) \\
\hline Camera 1 & 253159 & 256461 & -1.304 \\
Camera 2 & 251782 & 251766 & 0.006 \\
\hline \hline
\end{tabular}

Notes: Error is related to the difference between the convex hull area of a real and simulated acquisition.

obtained from the images shown in Fig. 6 are reported in Table I. It is possible to observe that the difference between the convex hull area of real and simulated samples is around $1 \%$. This value can be considered as satisfactory for this kind of application and it is mainly related to errors in the measurement of the acquisition setup and camera calibration.

In order obtain a quantitative measurement of the similarity between real and simulated data, we also computed the error obtained by estimating the convex hull area of the wood strand with a basic three-dimensional reconstruction technique [21]. The error have been computed considering the area of the scanned image as reference value. This error can be considered as adequate for this kind of measurement application, proving the accuracy of the proposed method in the shape simulation. In the case of the sample shown in 6, for example, the convex hull area computed from the scanned sample is equal to $15777 \mathrm{~mm}^{2}$ and the area of the three-dimensional model estimated from the synthetic images is equal to $16321 \mathrm{~mm}^{2}$, with an error of $3.45 \%$.

In real applicative scenarios, the measurement accuracy of three-dimensional reconstruction methods can be drastically reduced due to occlusions, noise, dust, and segmentation errors. For this reason, it should be necessary to adopt robust segmentation techniques [22] and specifically designed measurement methods [23-25].

Another performed test consists in the evaluation of the visual realism of synthetic images describing more complex simulated scenes acquired in industrial applications. Examples of obtained pairs of images are shown in Fig. 7. It is possible to observe that the reported images present satisfactory realism.

Finally, we have visually evaluated the realism of images obtained from a higher distance to the camera, considering a simulated space $S$ with $x \in[-95 \mathrm{~mm} \ldots 95 \mathrm{~mm}], y \in$ $[-95 \mathrm{~mm} \ldots 95 \mathrm{~mm}], z \in[750 \mathrm{~mm} \ldots 950 \mathrm{~mm}]$. Fig. 8 shows a frontal view of the performed simulation, which can be considered as sufficiently realistic.

The realism of the constructed scenes can be enhanced by including real conditions often present in the applications we want to simulated, such as: presence of dust in the volume; diffusion effects; reflections of the illumination system on metal parts; blur effect due to motion of the particles; aberrations and blur effects of the lenses. This kind of effects can be easily simulated by using tools like Blender [17] and Autodesk 3DS MAX [18]. In these software applications, however, it is particularly complex to simulate the complex and irregular shapes of wood strands. 
(a)

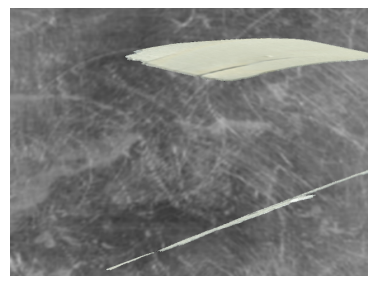

(c)
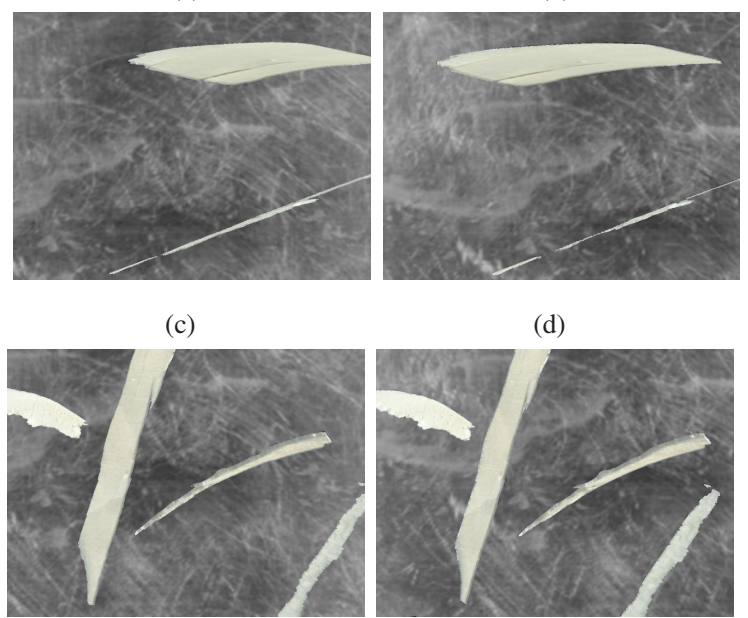

(d)

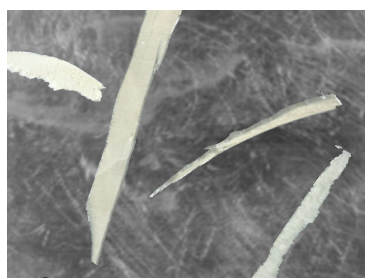

Fig. 7. Examples of samples simulating the inputs of a two view image processing system with a complex illuminated background simulating a dirt inox steel plate acquired in similar real conditions. The images are related to: (a) Camera 1 of the simulation A; (b) Camera 2 of the simulation A; (c) Camera 1 of the simulation B; (d) Camera 2 of the simulation B.

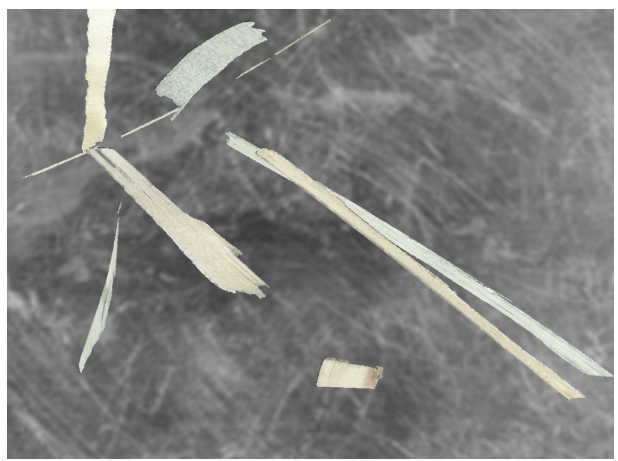

Fig. 8. Simulated image captured by Camera A and obtained from a virtual space $S$ with $z \in[750 \mathrm{~mm}$. . 950mm]. The simulated setup is equivalent to real experimental conditions.

\section{CONCLUSiON}

This paper has proposed a virtual environment for the computation of synthetic three-dimensional scenes describing free falling wood strand. The realized virtual environment permits to reduce the effort necessary for capturing big sets of samples with different hardware configurations in the design of measurement systems based on multiple view techniques. The implemented method, in fact, can simulate images captured by different viewpoints and particles with known size properties.

Experiments showed that the realized environment can obtain realistic samples and can effectively be used for testing three-dimensional measurement methods.

The proposed method, however, cannot completely substitute the use of real data for testing image processing systems for the particle size measurements, but it permits to reduce the impact of the first time-consuming and expensive steps in the design and preliminary evaluation of measurement algorithms since it can produce realistic data in a fast and repeatable manner.

\section{ACKNOWLEDGEMENTS}

This work has been partially supported by the EU within the 7FP project "Innovative Poplar Low Density Structural Panel (IPAN)" under grant agreement 308630. The IPAN project is coordinated by IMAL Srl (San Damaso, Modena, Italy).

\section{REFERENCES}

[1] International Organization for Standardization, "Iso 19.120, particle size analysis. sieving."

[2] R. L. Geimer and C. Link, Flake classification by image analysis. U.S Dept. of Agriculture, Forest Service, Forest Products Laboratory, 1988.

[3] T. Nishimura, J. Amin, and M. Ansell, "Image analysis and bending properties of model osb panels as a function of strand distribution, shape and size," Wood Science and Technology, vol. 38, pp. 297 - 309, 2004.

[4] V. Gaete-Martinez and S. M. Shaler, "Influence of log temperature in irregularities on strand geometry detected by digital image analysis," Maderas. Ciencia y tecnologÃa, vol. 9, pp. 211 - 221, 2007.

[5] S. Ferrari, V. Piuri, and F. F. Scotti, "Image processing for granulometry analysis via neural networks," in Proc. of the IEEE International Conference on Computational Intelligence for Measurement Systems and Applications (CIMSA 2008), July 2008, pp. 28 - 32.

[6] H. G. Merkus, Particle Size Measurements, 2009.

[7] S. Ferrari, V. Piuri, and F. Scotti, "Virtual environment for granulometry analysis," in Proc. of the IEEE Conf. on Virtual Environments, HumanComputer Interfaces and Measurement Systems, 2008, pp. 156 - 161.

[8] H. Zhang and M. Jägersand, "Granulometry using image transformation techniques," in Proc. of the 15th Int. Conf. on Vision Interface, 2002.

[9] J. Shu, L. T. Watson, B. G. Zombori, and F. A. Kamke, "Wbcsim: an environment for modeling wood-based composites manufacture," Engineering with Computers, vol. 21, no. 4, pp. 259 - 271, June 2006.

[10] M. López, J. M. Matías, J. A. Vilán, and J. Taboada, "Functional pattern recognition of $3 \mathrm{~d}$ laser scanned images of wood-pulp chips," in Pattern Recognition and Image Analysis, ser. Lecture Notes in Computer Science. Springer Berlin Heidelberg, 2007, vol. 4477, pp. 298 - 305.

[11] A. Kuhl, L. Kruger, C. Wohler, and U. Kressel, "Training of classifiers using virtual samples only," in Proc. of the 17th Int.Conf. on Pattern Recognition, vol. 3, 2004, pp. 418 - 421.

[12] R. Donida Labati, A. Genovese, V. Piuri, and F. Scotti, "Wildfire smoke detection using computational intelligence techniques enhanced with synthetic smoke plume generation," IEEE Trans. on Systems, Man and Cybernetics: Systems, vol. 43, no. 4, 72013.

[13] Y. Tan, X. Zhang, C. Wang, and Q. Zhao, "Real-time snowing simulation based on particle systems," in Proc. of the 1st Int. Workshop on Education Technology and Computer Science, vol. 3, 2009, pp. 7 - 11 .

[14] D. Nkulikiyimfura, J.-I. Kim, and H. Kim, "A real-time sand simulation using a gpu," in Proc. of the 8th Int.Conf. on Computing Technology and Information Management (ICCM), vol. 1, 2012, pp. 495 - 498.

[15] S. R. Buss, 3D Computer Graphics: A Mathematical Introduction with OpenGL. New York, NY, USA: Cambridge University Press, 2003.

[16] S. J. Gortler and R. I. Goldman, Foundations of $3 D$ computer graphics. University Press Group Limited, 2012.

[17] Blender, http://www.blender.org/.

[18] Autodesk, 3DS MAX, http://www.autodesk.com.

[19] R. C. Gonzalez and R. E. Woods, Digital Image Processing (3rd Edition). Upper Saddle River, NJ, USA: Prentice-Hall, Inc., 2006.

[20] R. I. Hartley and A. Zisserman, Multiple View Geometry in Computer Vision, 2nd ed. Cambridge University Press, 2004.

[21] R. Donida Labati, A. Genovese, V. Piuri, and F. Scotti, “Two-view contactless fingerprint acquisition systems: a case study for clay artworks," in Proc. of the IEEE Workshop on Biometric Measurements and Systems for Security and Medical Applications (BioMS), 9 2012, pp. 1 - 8.

[22] O. Wirjadi, "Survey of 3D Image Segmentation Methods," 2007.

[23] M. Kempkes, T. Vetter, and M. Mazzotti, "Measurement of 3d particle size distributions by stereoscopic imaging," Chemical Engineering Science, vol. 65, no. 4, pp. 1362 - 1373, 2010.

[24] E. Castillo-Castaneda and C. Turchiuli, "Volume estimation of small particles using three-dimensional reconstruction from multiple views," in Proce. of the 3rd Int. Conf. on Image and Signal Processing, ser. ICISP '08, 2008, pp. $218-225$.

[25] R. Donida Labati, A. Genovese, V. Piuri, and F. Scotti, "Low-cost volume estimation by two-view acquisitions: A computational intelligence approach," in Proc. of the 2012 IEEE-INNS International Joint Conference on Neural Networks (IJCNN 2012), 6 2012, pp. 1092 - 1099. 